\title{
Research on the Power and the Admission Control based on the Priority Mechanism in the Cognitive Radio
}

\author{
Zeyu Sun and Chuanfeng $\mathrm{Li}^{*}$
}

\author{
Computer and Information Engineering, Luoyang Institute of Science and Technology, Luoyang 471023, China
}

\begin{abstract}
Cognitive Radio is the new technology which can optimize the utilization of the frequency spectrum. The Primary User and the Secondary User utilize the same frequency spectrum so that the Signal-to- interference- plus-noiseratio of the Primary User will be reduced. In order to ensure the Quality-of-Service of the Primary User, the Power Control should be done to the Secondary User and the Admission Control should be combined to maximize the number of the Secondary User. In order to ensure the Quality-of-Service of the Secondary User, the Priority Mechanism is adopted to avoid the transmission interruption of the Secondary User. The simulated results show that the system performance is superior to the original algorithm.
\end{abstract}

Keywords: Bending, distortion, matlab, the center point, tilt.

\section{INTRODUCTION}

The request of the transmission quality by the wireless service industry based on the popularization of the modern mobile communication and the wireless network is more and more high [1]. The problem of the lack of the frequency spectrum is suffered under the environment of the limited frequency spectrum resources $[2,3]$. According to the research reports published by the Federal Communications Commission and the Defense advance Research Projects Agency; the utilization of the frequency spectrum has no sophisticated project and no high efficiency. Therefore, the Cognitive Radio is widely discussed and becomes the novel technology of improving the utilization of the frequency spectrum resources. The mainly concept is to register the frequency band's Spectrum Holes with the cognitive surveillance so that more Secondary Users cam transmit the data for reaching a higher utilization of the frequency spectrum [4].

The frequency Spectrum policy working group of FCC published a policy of allowing the unlicensed device to do the transmission in the television band in 2004. Its purpose was to build a wireless network on the basis of the Cognitive Radio in the television band without influencing the television service quality [5]. In order to make the wireless network operate in the television band, the first overcame problem is to know how the cognitive users can avoid causing the serious interference to the Primary User and influencing the service quality of the Primary User $[6,7]$. In the meantime, the cognitive users must maintain a certain service quality. The cognitive wireless base station should be erected for collecting each cognitive user's Channel State Information.
The base station should do the output power control to the cognitive users through these collected data, and the limited SINR should be set up to improve the whole service quality. Meanwhile, the admission control of the cognitive users through the base station in a certain limitation of the service quality should be conducted for make more cognitive users do the transmission.

The paper adapts to the Linear Programming Deflation algorithm in the Joint Admission and Power Control for Adhoc and Cognitive Underlay Networks: Convex Approximation and Distributed Implementation [8]. The LPD algorithm combines the power control with the admission control for reaching to the approaching optimal number of the Secondary Users. The removal procedure can cause the transmission interruption of the Secondary User. Therefore, the Linear Programming Deflation with Distance Priority algorithm and the Programming Deflation with Interference Priority algorithm are proposed in the paper to improve the number of the Secondary User during the period of ensuring the transmission of the cognitive users whose priority is rather high can not be interrupted and improving the number of the Secondary Users [9].

\section{THE SYSTEM FRAMEWORK OF THE COGNI- TIVE RADIO}

\subsection{The Cognitive Radio Network}

The Cognitive Radio Network is divided into two groups: the network of the Primary User. The Primary User has the priority of having the channel storage in the Primary User network, and it is just managed by the base station of the Primary User. Other non-authorized users can not cause excessive interference to it for it has the priority of having the channel storage [10]. 
The Cognitive Radio Network: The Cognitive Radio Network is called the non-authorized network and the dynamic frequency spectrum storage network. The Cognitive Radio has no frequency spectrum storage priority in the authorized channel so that the Cognitive Radio base station should be erected to offer the connection among the cognitive users. The Spectrum brokers should be set up to coordinate the frequency spectrum distribution of the cognitive users in the environment.

\section{The Power Control}

The power control has a great influence on the whole system efficiency in the Cognitive Radio System so that a better power control can improve the connection problem of the Cognitive Radio environment. Therefore, the power control does not only reach to the SINR limitation required by the cognitive users, but also avoids the excessive interruption to the Primary User. The power control in the Cognitive Radio is divided into two types: the centralized power control method and the distributed power control method. The power control in the television band will be introduced. In order to solve the interrupted problem encountered in the television band, the service quality between the cognitive users and the Primary Users is represented as SINR. The Primary User and the Secondary User can communicate the information detected from the environment. If there are $\mathrm{k}$ should be transmitted by the Secondary User, their transmitted power is $\mathrm{Pi}, \mathrm{sec}$, and SINR is $\mathrm{Yi}$,sec. The maximum efficiency and the problem of interrupting the power control can be shown as follows:

$$
\min \sum_{i=1}^{N} p_{i, \mathrm{sec}}
$$

Subject to $\gamma_{m, T V} \geq \gamma_{T V}^{\text {tar }}, \forall_{m}$

$\gamma_{i, \mathrm{sec}} \geq \gamma_{i, \mathrm{sec}}^{\mathrm{tar}}, i=1, \ldots, N$

$p_{\mathrm{sec}}^{\mathrm{min}} \leq p_{i, \mathrm{sec}} \leq p_{\mathrm{sec}}^{\mathrm{max}}, i=1, \ldots, N$

in which $\gamma_{T V}^{t a r}$ and $\gamma_{i, \mathrm{sec}}^{\mathrm{tar}}$ is the target SINR of the Primary User and the Secondary User, while $p_{\mathrm{sec}}^{\min }$ and $p_{\mathrm{sec}}^{\max }$ is the maximum and the minimum transmitted power of the transmitting terminal in the Secondary User. The maximal purpose of the power control in the Cognitive Radio is to maximize the energy efficiency and restrain the interruption to other users. The formula (2.1) can reach to the purpose, and the service quality of the Primary User and the Secondary User can be guaranteed by adding the formula (2.2) and the formula (2.3).

\subsection{The Admission Control}

When the number of the Primary User is too many and even exceeds the receptive number by the network, the network will be congested. At this time, the admission control should be required to control the number of the users. Eliminating the Secondary User whose inference is very large can guarantee the service requests of other users. In the paper, the Secondary User base station must collect the users' information. If the inference to the Secondary User is larger than the threshold of the Primary User, the Secondary User is not allowed to transmit the data transmission. How to allow the Secondary User to reach the systems maximum efficiency in the transmission is the mainly task of the Secondary User base station. When the admission control is done, more Secondary Users are expected to do the transmission simultaneously. The problem is as shown by the following equation:

$$
\begin{aligned}
& S_{0}=\arg \max _{s \in\{1, \ldots k\},\{p k \in R+\}_{k}^{k}=1}|S| \\
& \text { subject to } p_{k} \leq P_{k}^{M A X}, \forall k \in\{1, \ldots K\} \\
& \frac{G_{k k p k}}{\sum_{l=1, l \neq k}^{K} G_{l k p l}+G_{o k p o+\sigma_{k}^{2}}} \geq C_{k}, \forall k \in S_{0} \\
& \frac{G_{00} p_{0}}{\sum_{l \in S_{0}} G_{l 0}+\sigma_{0}^{2}} \geq C_{0}
\end{aligned}
$$

in which $|S|$ is the number of set S's element, the formula (2.7) is the SINR limitation of the Secondary User, the formula (2.8) is the SINR limitation of the Primary User, $P_{k}^{\text {MAX }}$ is the limitation of the maximum transmission power of the Secondary User, $G_{k k}$ is the channel gain of the Secondary User $\mathrm{k}, G_{l k}$ is the channel gain from other Secondary Users to the Secondary User k, $G_{00}$ is the channel gain of the Primary User, $G_{l 0}$ is the channel gain from Secondary User to the receiving terminal of the Primary User, $G_{0 k} p_{0}$ is the interruption from the Primary User to the Secondary User k, $\sigma_{0}^{2}$ and $\sigma_{k}^{2}$ is the noise from the receiving terminal of the Primary User to the receiving terminal of the Secondary User. The selection of the sun set to the Secondary User is a NP-hard problem.

\subsection{The Combination of the Power Control and the Ad- mission Control}

The paper should consider the power control and the admission control simultaneously for optimizing the number of the Secondary Users. At this time, the following conditions must be satisfied: 1 . The Primary User must guarantee the superior service quality: 2. If the Secondary Users is transmitted by the admission, it must also have the superior service quality: 3 . When the number of the Secondary Users transmitted by the admission is maximized, the service of the power consumption in these Secondary Users should be minimized. 


\section{CONVEX APPROXIMATION AND ITS ALGO- RITHM}

\subsection{The Signal Layer Integration}

The chapter adapts to the application of the convex approximation in the paper [2,3] and [5]. As the power control and the admission control can has the operated formula respectively and both of them should be combined so that the equation should be rewrote. The problems of the two layers between the (2.1) (2.4) and the (2.5) (2.9) can be integrated into a optimization problem of a signal layer which has the equal efficiency. How to solve the following problems should be considered:

$$
\begin{aligned}
& \min _{\left\{p_{k \in R+s k \in(-1,+1)}\right\}_{k=1}^{K}} \in \sum_{k=1}^{K} p k+(1-\in) \sum_{k=1}^{K} \lambda k(s k+1)^{2} \\
& \text { subject to } p_{k} \leq P_{k}^{M A X}, \forall k \in\{1, \ldots K\} \\
& \frac{G_{k k} p_{k}+\delta_{k}^{-1}\left(s_{k}+1\right)^{2}}{\sum_{l=1, l \neq k}^{K} G_{l k} p_{l}+G_{o k} p_{o}+\sigma^{2}} \geq c_{k}, \forall k \in\{1, \ldots, K\} \\
& \frac{G_{k k} p_{k}+\delta_{k}^{-1}\left(s_{k}+1\right)^{2}}{\sum_{l=1}^{K} G_{l 0} p_{l}+\sigma_{0}^{2}} \geq c_{0}
\end{aligned}
$$

The binary scheduling variable $s_{k}$ should be added in the formula. When its value is -1 , the Secondary User can be allowed to do the transmission. When its value is 1 , the Secondary User can not be allowed to do the transmission. When the value of the $\delta_{0}$ is small and the $\delta_{0}$ is 1 , the k Secondary User can not do the transmission when the k Secondary User can not reach to the SINR limitation. When $s_{k}$ is -1 , the data can be transmitted when the $\mathrm{k}$ Secondary User reaches to the SINR standard. The formula (3.1) includes the power control and the admission control and the discrete value represents the part of consumption in the admission control. The consumption value in the part of the power control has the bounded value with the limitation of the formula (3.2). When $\varepsilon$ is set as a small value, the admission control is guaranteed to be superior to the power control for improving the accuracy of selecting the number of the Secondary User.The following result can be obtained with the CauchySchwartz inequality.

$$
\begin{aligned}
& \text { For } \lambda_{k}=1, \forall k \in\{1, \ldots, K\}, \text { and } 0<\varepsilon<\frac{4}{\sum_{k=1}^{K} P_{k}^{M A X}+4} \\
& \delta_{k}<\frac{4}{c_{k}\left(\sum_{l=1, l \neq k}^{K} G_{l k} P_{l}^{M A X}+G_{0 k} p_{0}+\sigma_{k}^{2}\right)}
\end{aligned}
$$

Solving the formula (3.1) (3.4) is equal to solve the formula (3.1) (3.4). In fact, when the formula (3.1) (3.4) has many solutions, a group of the minimum power can be produced when the formula (3.1) (3.4) is solved, and its linear deduction is similar to the reference [5]. The variable $\lambda \mathrm{k}$ is added in the formula to judge whether it can be used as the weighted value for improving its fairness or the social welfare. When the set $\lambda \mathrm{k}$ is proportionally distributed to the queuing length of the $\mathrm{k}$ Secondary User and the system output can be optimized [8]. If it is reversed to the users' service quality, its fairness will be improved. When the $\lambda$ of the Secondary User is not equal, the formula (2.1) (2.4) and the formula (2.5) (2.8) will loss balance. Therefore, the formula (3.1) (3.4) is still a NP -Hard problem.

\subsection{Isolating Non-Convexity}

The problem of the formula (3.1) (3.4) is not directly accorded with the convex approximation so that the formula must be rewrote and a part of the isolating non-convexity should be clearly displayed. The optimized formula can be obtained.

$$
\begin{aligned}
& \left\{p k \in R_{+, s k \in R^{2 \times 2}}^{\min }\right\} \varepsilon \sum_{k=1}^{K} p k+(1-\varepsilon) \sum_{k=1}^{K} \lambda_{k} \operatorname{Tr}\left(1_{2} \times_{2} S_{k}\right) \\
& \text { subject to } p k \leq p_{k}^{M A X}, \forall k \in\{1, \ldots K\} \\
& \frac{G_{k k} p_{k}+\delta_{k}^{-1} \operatorname{Tr}\left(1_{2} \times_{2} S_{k}\right)}{\sum_{l=1, l \neq k}^{K} G_{l k p_{l}}+G_{o k} p_{o}+\sigma_{k}^{2}} \geq c_{k}, \forall k \in\{1, \ldots K\} \\
& \frac{G_{o o} p_{o}}{\sum_{l=1, l \neq k}^{K} G_{l o} p_{o}+\sigma_{o}^{2}} \geq c_{o} \\
& S_{k} \geq 0, \operatorname{rank}\left(S_{k}\right)=1, S_{k}(1,1)=S_{k}(2,2)=1 \\
& \forall k \in\{1, \ldots K\} \\
& \left(S_{k}+1\right)^{2}=\left(\left[\begin{array}{ll}
s_{k} & 1
\end{array}\right]\left[\begin{array}{l}
1 \\
1
\end{array}\right]\right)^{2} \\
& =\operatorname{Tr}\left(\left[\begin{array}{ll}
1 & 1 \\
1 & 1
\end{array}\right]\left[\begin{array}{l}
S_{k} \\
1
\end{array}\right]\left[\begin{array}{ll}
s_{k} & 1
\end{array}\right]\right)=\operatorname{Tr}\left(1_{2 \times 2} S_{k}\right)
\end{aligned}
$$

$\operatorname{Tr}(\cdot)$ is the trace of the matrix, $S_{k}=S_{k} S_{k}{ }^{T}$ 和 $S_{k}:=\left[s_{k} 1\right]^{T}$.

$\mathrm{Sk} \geq 0$ is the Positive semi definite matrix in the matrix $\mathrm{Sk}$. The value of the diagonal element is ', and the value of the non diagonal element remains the original set parameter sk. The matrix $12 \times 2$ is a unit matrix $2 \times 2$. The Sk just can have two possibilities under the condition of $\operatorname{rank}(\operatorname{Sk})=1, \operatorname{Sk}(1,1)=\operatorname{Sk}(2,2)=1$ :

$$
\begin{aligned}
& S_{k}=\left[\begin{array}{ll}
1 & 1 \\
1 & 1
\end{array}\right] \rightarrow \operatorname{Tr}\left(1_{2 \times 2} S_{k}\right)=4 ; \text { or } \\
& S_{k}=\left[\begin{array}{rr}
1 & -1 \\
-1 & 1
\end{array}\right] \rightarrow \operatorname{Tr}\left(1_{2 \times 2} S_{k}\right)=0
\end{aligned}
$$

When the value of the rank is limited as 1 , the $\mathrm{Sk} \in\{-$ $1,1\}$ can be obtained, which is the only source of making the formula (3.7) (3.11) become the isolated non convexity.

\subsection{Semi Definite Programming Relaxation}

When the limitation of rank $=1$ is removed, the representative $S_{k}$ can get the value between $[-1+1]$ and the 
problem of the Semi definite Programming can be obtained. The description of the rank relaxation in the reference [9] can cause a problem of the Lagrangian bi-dual which is the easiest way to solve the formula (3.7) (3.11). Aiming to the real symmetric matrix $2 \times 2 S_{k}$, its unit diagonal element can be wrote as $\left[\begin{array}{cc}1 & x_{k} \\ x_{k} & 1\end{array}\right]$. When there is a $\mathrm{xk} \in\{-1,1\}$, the value of the two row vectors is in proportion. When the determinant is the non negative value and its necessary and sufficient condition is $\mathrm{xk} \in\{-1,1\}$. The matrix $S_{k}$ in the

formula (3.7) (3.11) is started through the $t_{k}:=\operatorname{Tr}\left(1_{2 \times 2} S_{k}\right)$ and the parametric value $t_{k}=2\left(1+x_{k}\right)$. The limitation of rank $=1$ can be deleted, the limited range $[-1,1]$ can be converted into the equal efficient parameter tk, and its limited range is $[0,4]$. The following linear formula can be obtained as follows:

$$
\begin{gathered}
\min _{\left\{p k \in R+, S k \in R^{2 \times 2}\right\}} \varepsilon \sum_{k=1}^{K} p k+(1-\varepsilon) \sum_{k=1}^{K} \lambda_{k} t_{k} \\
\text { subject to } p_{k} \leq P_{k}^{M A X}, \forall k \in\{1, \ldots, K\}
\end{gathered}
$$

$\frac{G_{k k} p_{k}+\delta_{K}^{-1} t_{k}}{\sum_{l=1, l \neq k}^{K} G_{l k} p_{l}+G_{o k} p_{o}+\sigma_{k}^{2}} \geq c_{k}, \forall k \in\{1, \mathrm{~K}, K\}$

$\frac{G_{o o} p_{o}}{\sum_{l=1, l \neq k}^{K} G_{l o} p_{o}+\sigma_{o}^{2}} \geq c_{o}$

$0 \leq t_{k} \leq 4, \quad \forall k \in(1, \ldots, K)$

When we solve the problem of the formula (3.13) (3.17), the lower bound in the formula (3.7) (3.11) can be obtained from the produced solutions so that the sub optimal solution in the formula (3.7) (3.11) can be obtained.

\subsection{The Priority Mechanism Algorithm}

The mainly idea is to reduce the number of the possible solution found by the Secondary User in the formula (3.13) (3.17). When we solve the formula (3.13) (3.17), whether the SINR of the whole Primary User and the whole Secondary User meet the requirement of the limitation should be confirmed. It not, the Secondary User which has a lower priority should be selected so that the transmission can not be done. The above steps should be repeated until all users meet the requirement of the limitation.

Algorithm 1: Linear Programming Deflation with Distance Priority

1. There is a set $U$ of the Secondary User to do the transmission, $U \leftarrow\{1, \cdots, K\} .2$. The Secondary User base station gives the priority from near to far based on the previous prior marks and the distance between other Secondary Users and the base station. The nearer distance of the Secondary User has a higher priority. The new set $\mathrm{Ud} \leftarrow$ $\{1 \mathrm{~d}, \cdots, \mathrm{K} \mathrm{d}\}$ after the ordering can be obtained. 3 .
The Secondary User within the set Ud should be operated in the formula (3.13) (3.17). 4. If all Secondary Users can reach to the target SINR value, the operation can be interrupted. If the SINR value of the Secondary User does not meet the requirements, it should be judged from the Secondary User which has a higher priority. It it meets the requirements, the next Secondary User should be judged. If not, the Secondary User combination of the maximal inference value Glkpl should be eliminated.

Algorithm 2: Linear Programming Deflation with Interference Priority

1. The Secondary User in the set $U$ requires transmission, $\mathrm{U} \leftarrow\{1, \cdots, \mathrm{K}\}$.

2. The Secondary User base station sorts the size of the receiving terminal inference G10pl in the Primary User based on the previous prior marks and the distance between other Secondary Users and the base station. The smaller the inference is, the higher the priority of the Secondary User has. The new set $\mathrm{U} 1 \leftarrow\{11, \cdots, \mathrm{K} 1\}$ can be obtained.

3. The Secondary User within the set U1 should be operated in the formula (3.13) (3.17). 4. If all Secondary Users can reach to the target SINR value, the operation can be interrupted. If the SINR value of the Secondary User does not meet the requirements, it should be judged from the Secondary User which has a higher priority. It it meets the requirements, the next Secondary User should be judged. If not, the Secondary User combination of the maximal inference value Glkpl should be eliminated.

The difference between the algorithm 1 and the algorithm 2 is the accordance defined by the priority. The algorithm 1 is according to the distance between the Secondary User and the Secondary User base station, while algorithm 2 is according to the inference value size from the Secondary User to the Primary User. The paper proposes two methods aiming to how the Secondary User base station does the eliminating movement to the priority of the Secondary User. 1. Relative Priority: The Secondary User base stations with meet the requirements of a higher priority of the Secondary User, while other Secondary Users which has a lower priority can be deleted. 2. Absolute Priority: When the SINR value in the users which have a higher priority can not reach to the target, the the Secondary User base station can eliminate the Secondary User which has a lower priority.

\section{SIMULATION AND ANALYSIS}

The simulated environment used in the paper is the network framework of the Cognitive Radio, as shown in the Fig. (1). If the Secondary User base station has the complete channel state information and the region of the Cognitive Radio is the square region whose space is 2 kilometers, there is a Secondary User base station of the region's center and a Primary User receiving terminal in the 2 kilometers from the television base station. The minimal power $P_{k}^{M I N}$ transmitted by the cognitive users should be meet the requirements of the needed power of the SINR limitation without other interruptions of other Secondary Users. The power budget of the 


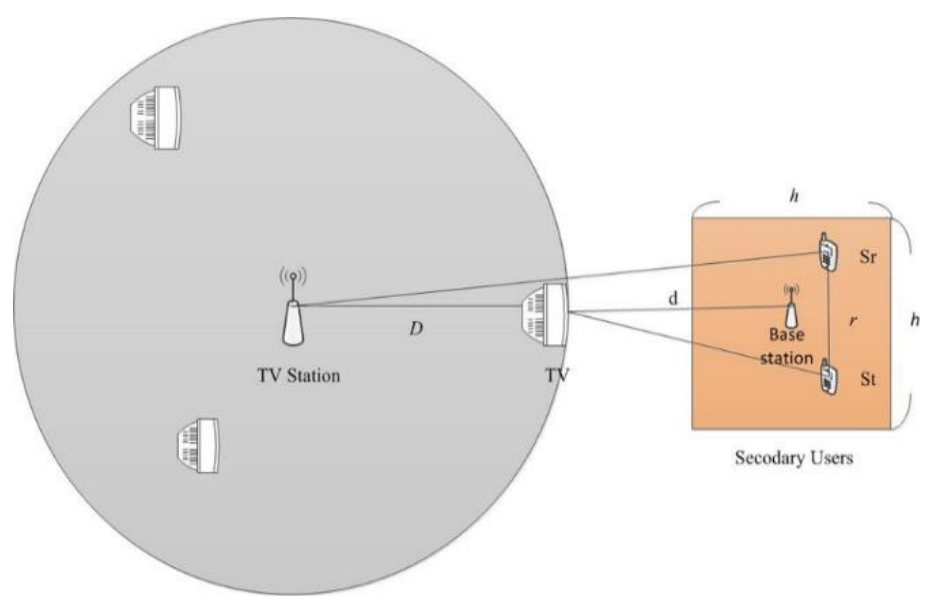

Fig. (1). The cognitive radio is operated in the television band.

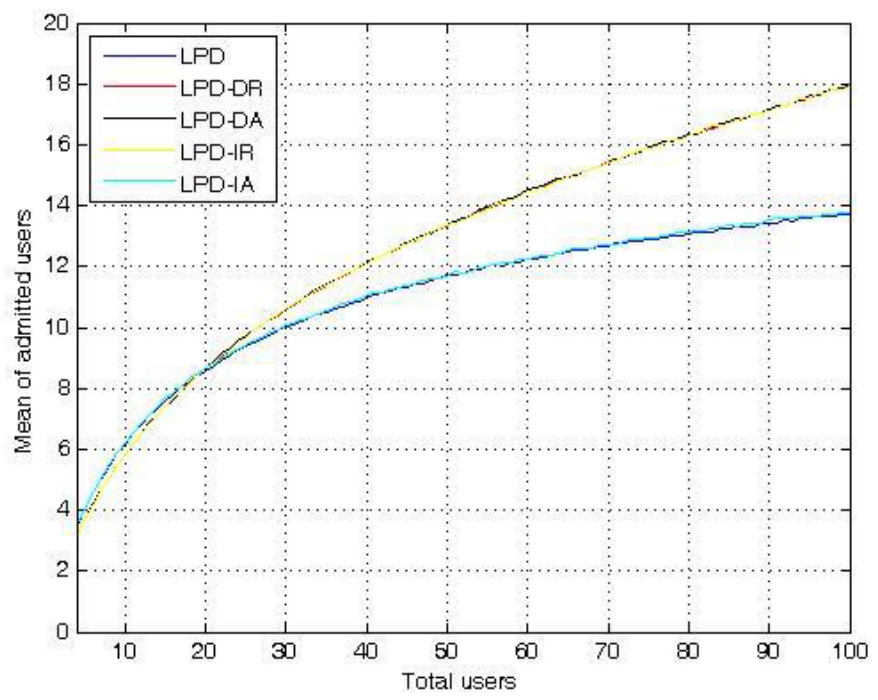

Fig. (2). The compared figure of the secondary user.

Secondary User can be represented as follow: $P_{k}^{M A X}=b P_{k}^{M I N}$. The Primary User whose transmission power is $100 \mathrm{kw}$ does the television signal transmission, and the channel gaining can be represented as follows: $G_{i j}=1 / d_{i j}^{a} \cdot d_{i j}^{a}$ is the Euclidean distance between the transmitting terminal $i$ and the receiving terminal $\mathrm{j}$. a is the index of the path loss because the television transmitting terminal is designed by a high antenna so that the cognitive users are in the ground. Therefore, the cognitive users are faster than the television transmitting terminal at the distance loss aspect. If the loss index of the television base station is set as 3 , the loss index of the Primary User environment is set as 4 .

As to the relaxation algorithm, the $\sigma_{\varepsilon}$ should be multiplied 0.999 , while $\varepsilon$ is set as the one over ten of the original Table 1.

The SINR value of the Primary User in the Fig. (2) is set as $2 \mathrm{~dB}$ and the SINR value of the Secondary User is set as
$2 \mathrm{~dB}$. D represents the distance; as stands for the inference and a means the absolute screening method. The figure shows that the relative screening method and the absolute screening method has no great influence on the system performance in which the distance is used as the LPD of the priority. Therefore, the absolute priority should be directly selected to guarantee the users who have a higher priority not to interrupt the transmission. If the Secondary User is less 20 in the algorithm which uses the inference as the priority, the performance of the absolute priority is better. If the Secondary User is over 20 , the performance of the relative priority is better. Therefore, the system can adapt to the absolute priority screening method when the number of the Secondary User is less 20. When the number is over 20, the relative priority screening method is adopted and the number of the mean of admitted users increase more 4 than the original algorithm. In this way, the whole performance of the system can be improved and the number of the Secondary User allowed doing the transmission by the base stations can be 
Table 1. Model parameters.

\begin{tabular}{|c|c|}
\hline $\mathbf{h} \times \mathbf{h}$ & $\mathbf{2 \times 2}(\mathbf{k m})$ \\
\hline \hline $\mathrm{d}$ & $2(\mathrm{~km})$ \\
\hline $\mathrm{PTV}$ & $100 \mathrm{~kW}$ \\
\hline $\mathrm{R}$ & $70 \mathrm{~km}$ \\
\hline $\mathrm{r}$ & $400 \mathrm{~m}$ \\
\hline$\sigma 2$ & $-70 \mathrm{dBm}$ \\
\hline$\alpha \mathrm{TV}$ & 3 \\
\hline$\alpha \mathrm{k}$ & 4 \\
\hline $\mathrm{b}$ & 5 \\
\hline$\delta \mathrm{k}$ & $\times 0.999$ \\
\hline$\varepsilon$ & $\times 0.1$ \\
\hline
\end{tabular}

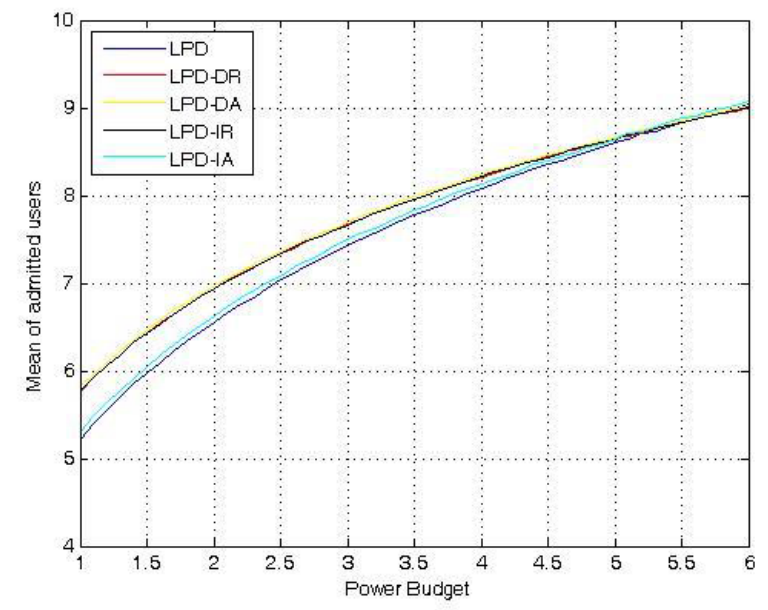

Fig. (3). The comparison of the power budget (The number of the Secondary User is 20).

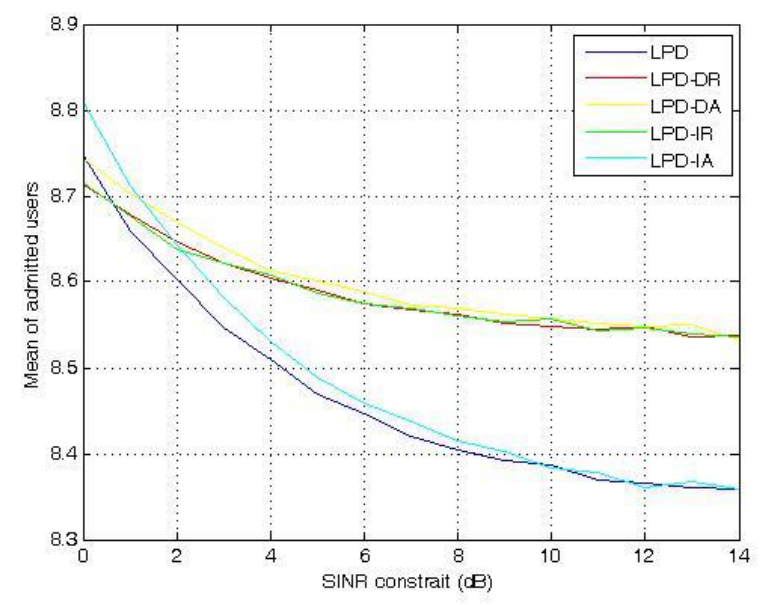

Fig. (4). The SINR comparison of the Secondary User (The number of the Secondary User is 20). 
increased under the condition of guaranteeing the transmission of the Secondary Users who have a higher priority not to be interrupted.

The Fig. (3) is the comparison of the power budget. When the power budget is higher, the bounded value of the transmission power in the Secondary User is larger. Therefore, the Secondary User can transmit a larger power so that the possibility of satisfying the SINR requirements can be improved.

The Fig. (4) shows that the number of the Secondary User allowed to do the transmission is gradually

Reducing when the SINR requirements of the Secondary User is higher.

\section{CONFLICT OF INTEREST}

The authors confirm that this article content has no conflict of interest.

\section{ACKNOWLEDGEMENTS}

This work is supported by the Science of Technology Research of Foundation Project of Henan Province Education Department under Grant Nos. 2014B520099, Natural Science and Technology Research of Foundation Project of Henan Province Department of Science under Grant Nos. 142102210471, Science and Technology Research Project Of Education Department of Henan Province (14A510009), the Funding Scheme for Youth Teacher of Henan Province (2012GGJS-191), the Science and Technology Key Project of Henan Province (142102210568, 142102210063). of the $5^{\text {th }}$ International Conference on Cognitive Radio Oriented Wireless Networks \& Communications, 2010, pp. 1-5.

[2] J. Unnikrishnan, and V. V. Veeravalli, "Algorithms for dynamic spectrum access with learning for cognitive radio, " IEEE Transactions on Signal Processing, vol. 58, no. 2, pp. 750-760, 2010.

[3] C. H. Jiang, J. K. Lain, R. M. Weng, and C. H. Hsu, " Multi-rate DS-CDMA with ANFIS-assisted power control for wireless multimedia communications," International Journal of Innovative Computing, Information and Control, vol. 6, no. 8, pp. 3641-3656, 2010.

[4] FCC, Spectrum Policy Task Force Report, ET Docket No. 02-155, 2002.

[5] I. Mitliagkas, N.D. Sidiropoulos, and A. Swami, "Joint power and admission control for ad-hoc and cognitive underlay networks: convex approximation and distributed implementation," IEEE Transactions on Wireless Communications, vol. 10, no. 12, pp. 4110-4121, 2011.

[6] I. Mitliagkas, N.D. Sidiropoulos, and A. Swami, "Convex approximation-based joint power and admission control for cognitive underlay networks," In: Wireless Communications and Mobile Computing Conference, 2008. IWCMC '08. International, 2008, pp. 28-32.

[7] J. Huang, H. Wang, Y. Qian, and C. Wang, "Priority-based traffic scheduling and utility optimization for cognitive radio communication infrastructure-based smart grid," IEEE Transactions on Smart Grid, vol. 4, no. 1, pp. 78-86, 2013.

[8] Y. Zhang, J. Zheng and H. H. Chen, Cognitive Radio Networks: Architectures, Protocols and Standards, Taylor \& Francis, Boca Raton, 2010.

[9] E. Matskani, N.D. Sidiropoulos, Z.Q. Luo, and L. Tassiulas, "Convex approximation techniques for joint multi user down link beam forming and admission control," IEEE Transactions on Wireless Communications, vol. 7, no. 7, pp. 2682-2693, 2008.

[10] Y. Zhang, R. Yu, M. Nekovee, Y. Liu, S. Xie, and S. Gjessing, "Cognitive machine-to-machine communications: visions and potentials for the smart grid," IEEE Network, vol. 26, no. 3, pp. 613, 2012.

\section{REFERENCES}

[1] H. Nam, M. B. Ghorbel, and M. Alouini, "Location-based resource allocation for OFDMA cognitive radio systems," In: Proceedings

Received: September 16, 2014

Revised: December 23, 2014

Accepted: December 31, 2014

(C) Sun and Li; Licensee Bentham Open.

This is an open access article licensed under the terms of the Creative Commons Attribution Non-Commercial License (http:/creativecommons.org/licenses/by$\mathrm{nc} / 3.0 /$ ) which permits unrestricted, non-commercial use, distribution and reproduction in any medium, provided the work is properly cited. 